\title{
Four-way Self-weighted Alternating Normalized Residue Fitting Algorithm with Application for the Analysis of Serotonin in Human Plasma
}

\author{
Ya-Juan LiU, Hai-Long Wu, ${ }^{\dagger}$ Chao KANG, Hui-Wen Gu, Jin-Fang NIE, Shan-Shan LI, \\ Zhi-Yi Su, and Ru-Qin Yu \\ State Key Laboratory of Chemo/Biosensing and Chemometrics, College of Chemistry and Chemical \\ Engineering, Hunan University, Changsha 410082, P. R. China
}

\begin{abstract}
A novel algorithm, four-way self-weighted alternating normalized residue fitting (SWANRF), which is an extension of its three-way form, for the decomposition of quadrilinear data with new weight factors, was proposed and applied to the quantitative analysis of serotonin contents in plasma samples. It was observed that the third-order calibration could not only retain a "second-order advantage" and but also obtain other advantages. The introduction of a fourth mode can relieve the serious problem of collinearity, which seems to be one of the "third-order advantages". The proposed algorithm shows great potential as a promising alternative for the third-order calibration of a four-way data array by contrasting with four-way parallel factor analysis (four-way PARAFAC). Furthermore, both algorithms mentioned above were utilized to analyze the 5-hydroxytryptamine (serotonin) contents in plasma samples by obtaining four-way array (excitation-emission-pH-sample) data, and produced satisfactory results. The serotonin contents in plasma samples obtained by using four-way SWANRF and four-way PARAFAC were $0.324 \pm 0.005$ and $0.348 \pm 0.006 \mathrm{nmol} \mathrm{mL}^{-1}$, respectively.
\end{abstract}

(Received May 22, 2012; Accepted September 13, 2012; Published November 10, 2012)

\section{Introduction}

Calibration is the mathematical and statistical process of extracting information, usually quantifying analyte(s), based on the obtained instrument signal. Along with the rapid development of analytical equipment, high-order calibration as a powerful tool is playing a significant role in the determination of components of interest, even in the presence of complex background or uncalibrated inteferences. ${ }^{1-3}$ Nowadays, "second-order calibration" should be considered as the most popular multi-way data analysis method; this includes, for instance, the generalized rank annihilation method (GRAM), ${ }^{4-6}$ parallel factor analysis (PARAFAC), ${ }^{7-10}$ alternating trilinear decomposition (ATLD), ${ }^{11}$ self-weighted alternating normalized residue fitting (SWANRF) ${ }^{12}$ and so on. ${ }^{13-18}$ They aim to search for any "second-order advantage" by three-dimensional responsive data; that is, second-order calibration has better stability towards interferents as well as matrix effects. Thus, they have been brought into extensive practical applications, for example, biological matrices, pharmaceuticals, food, and environment, ${ }^{19-27}$ while seeking out a way to quantify the analytes of interest, even in presence of complex background.

A multiway calibration method includes not just second-order calibration, but also third-order calibration, and even higher-order calibration. Theoretically, third-order calibration contains one advantage similar to "second-order advantage", and it holds

$\dagger$ To whom correspondence should be addressed.

E-mail: hlwu@hnu.edu.cn additional advantages. For example, with trilinear data for one sample, the intrinsic profiles in each mode could be determined uniquely for each species, which is considered as one of the "third-order advantages". ${ }^{28,29}$ Nevertheless, the complete "third-order advantage", or the Nth-order advantage, is still unknown totally. ${ }^{1}$ To the best of our knowledge, only a few algorithms, such as four-way PARAFAC, ${ }^{30,31}$ trilinear least-squares (TLLS), ${ }^{32}$ alternating penalty quadrilinear decomposition (APQLD), ${ }^{33}$ alternating weighted residue constraint quadrilinear decomposition (AWRCQLD), ${ }^{34}$ can be used for the third-order calibration of four-way data. Accordingly, to search for the "third-order advantage", more related studies on four-way data analysis and third-order calibration are worth further investigation.

In the present paper, four-way SWANRF, which is regarded as a novel extension of its three-way form, along with new weight factors, was developed. Both four-way SWANRF and four-way PARAFAC methods were employed in the third-order calibration of four-way data arrays, including simulated and real datasets. It was found that one of the "third-order advantages" is that the introduction of a fourth mode is capable to relieve the serious problem of collinearity to some extent. Further, four-way SWANRF for a four-way data array was proved to be a new algorithm to deal with third-order calibration.

Serotonin is well known as a neurotransmitter in the control and regulation of many brain functions. A variety of physiological processes, including smooth muscle contraction, blood-pressure regulation and both peripheral and central nervous system neurotransmission, are strongly implicated by serotonin. $^{35-40}$ Some methods, such as radioimmunoassay, ${ }^{41}$ 
Table 1 Nomenclature mentioned for detailed information

\begin{tabular}{|c|c|}
\hline$\underline{\mathbf{X}} q$ & Four-way data array \\
\hline$I, J, K, L$ & The dimensions of four modes of $\underline{\mathbf{X}} q$ \\
\hline$x_{i j k l}$ & The $i j k l$ th element of $\underline{\mathbf{X}} q$ \\
\hline $\mathbf{A}_{I \times N}, \mathbf{B}_{J \times N}, \mathbf{C}_{K \times N}, \mathbf{D}_{L \times N}$ & $\begin{array}{l}\text { The four underlying profile matrices of } \underline{\mathbf{X}} q \\
\text { with } I \times N, J \times N, K \times N, L \times N \text { respectively }\end{array}$ \\
\hline$a_{i n}, b_{j n}, c_{k n}, d_{j k}$ & $\begin{array}{l}\text { The } i n t h, j n \text { th, } k n \text {th and } l n \text {th element of the } \\
\text { four underlying profile matrices A, B, C and } \\
\text { D, respectively }\end{array}$ \\
\hline $\boldsymbol{a}_{(i)}, \boldsymbol{b}_{(j)}, \boldsymbol{c}_{(k)}, \boldsymbol{d}_{(l)}$ & $\begin{array}{l}\text { The } i \text { th, } j \text { th, } k \text { th and } l \text { th row vectors of profile } \\
\text { matrices } \mathbf{A}, \mathbf{B}, \mathbf{C} \text { and } \mathbf{D} \text {, respectively }\end{array}$ \\
\hline $\operatorname{diag}\left(\boldsymbol{a}_{(i)}\right), \operatorname{diag}\left(\boldsymbol{b}_{(j)}\right), \operatorname{diag}\left(\boldsymbol{c}_{(k)}\right), \operatorname{diag}\left(\boldsymbol{d}_{(\ell)}\right)$ & $\begin{array}{l}\text { Diagonal matrices with elements equal to the } \\
\text { elements of } \boldsymbol{a}_{(i)}, \boldsymbol{b}_{(j)}, \boldsymbol{c}_{(k)} \text { and } \boldsymbol{d}_{(l)} \\
\text { respectively }\end{array}$ \\
\hline $\mathbf{X}_{i . . l}, \mathbf{X}_{. . k l}, \mathbf{X}_{j k .}, \mathbf{X}_{i j . .}$, & The complete slices of $\underline{\mathbf{X}} q$, respectively \\
\hline $\mathbf{X}_{\ldots l}, \mathbf{X}_{i \ldots,}, \mathbf{X}_{j . .}, \mathbf{X}_{\ldots k}$ & The incomplete unfolding of $\underline{\mathbf{X}} q$, respectively \\
\hline $\mathbf{X}_{I \times J K L}, \mathbf{X}_{J \times K L L}, \mathbf{X}_{K \times L L J}, \mathbf{X}_{L \times I J K}$ & The incomplete slices of $\underline{\mathbf{X}} q$, respectively \\
\hline$\odot$ & Khatri-Rao product ${ }^{47}$ \\
\hline$\|\cdot\|_{F}$ & The Frobenius matrix norm; \\
\hline+ & $\begin{array}{l}\text { The Moore-Penrose generalized inverse of } \\
\text { matrices }^{48}\end{array}$ \\
\hline SSR & The sum of squared residuals \\
\hline $\mathbf{W}_{A}, \mathbf{W}_{B}, \mathbf{W}_{C}$ and $\mathbf{W}_{D}$ & Weight factors \\
\hline
\end{tabular}

enzyme immunoassay, ${ }^{42}$ gas chromatography, ${ }^{43,44}$ capillary electrophoresis-electrospray ionization-time-of-flight mass spectrometry, ${ }^{45}$ and high-performance liquid chromatography, ${ }^{46}$ have been used to analyze serotonin quantitatively. However, all of aforementioned methods based on "physical or chemical separation" need complicated pretreatment and expensive analytical instruments. Yet, four-way PARAFAC and the proposed method four-way SWANRF can be used for the direct quantitative analysis of serotonin contents in plasma samples with excitation-emission-pH-sample data, by means of "mathematic separation", which is inexpensive and labor-saving. Eventually, sensitivity (SEN) was estimated by a reliable method in this paper, gaining commendable results.

\section{Theory}

\section{Nomenclature}

The nomenclature applied throughout this paper is listed in Table 1 . The inner cyclic symmetry property exits in multilinear decomposition. ${ }^{15}$ The four-way cyclic symmetry of quadriclinear decomposition, which is a feature of four-way data, is an extension of the cyclic symmetry of trilinear decomposition. As visualized in Fig. 1, the physical modes, subscripts, elements and vectors in resolved matrices, resolved matrices, sliced matrices, incomplete unfolded matrices unfolded matrices, as well as residue and resolution formulas, all obey the inner cyclic symmetry property, circumrotating along the same way. These mathematical properties provide help to understand and develop multi-way calibration methods.

The proposed famous three-way trilinear component model ${ }^{7,10}$ places an important impact on the three-way data analysis. Similarly, according to the quadrilinear model, each element, $x_{\mathrm{ijk} k}$, of the data array $\mathbf{X} q$ can be represented as follows:

$$
\begin{aligned}
x_{\mathrm{ijkl}}= & \sum_{\mathrm{n}=1}^{\mathrm{N}} a_{\mathrm{in}} b_{\mathrm{jn}} c_{\mathrm{kn}} d_{\mathrm{ln}}+e_{\mathrm{ijkl}} \\
& (i=1,2, \ldots, I, j=1,2, \ldots, J, k=1,2, \ldots, K, l=1,2, \ldots, L) .
\end{aligned}
$$

The quadrilinear model can be expressed in four fully equivalent stretched matrix representations, respectively, owing
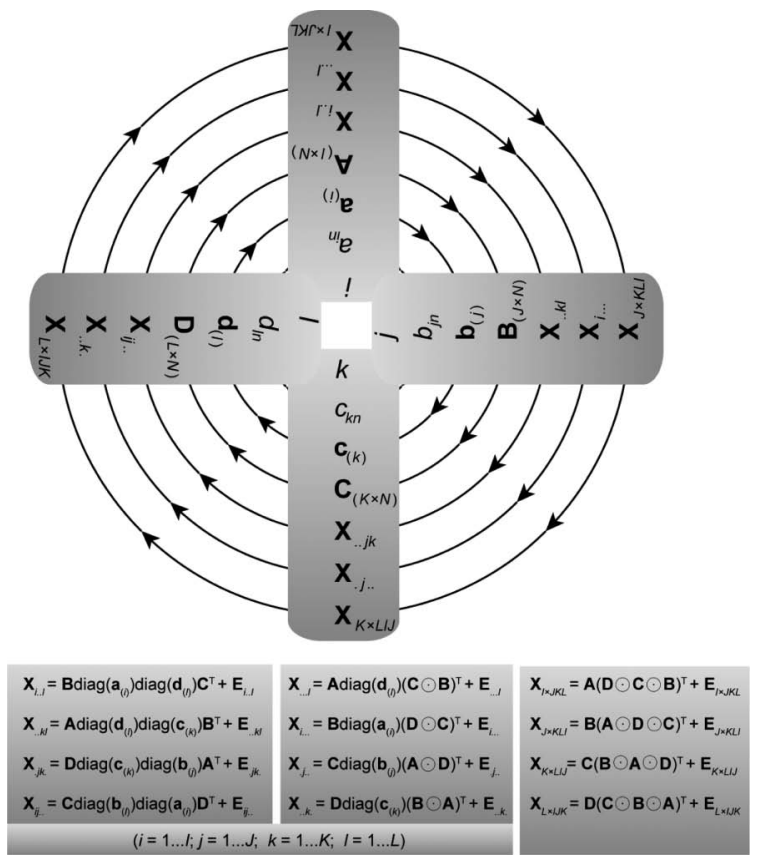

Fig. 1 Visualization of the property for the quadrilinear component model, named as a four-way cyclic symmetry property.

to the cyclic symmetry of the model as:

$$
\mathbf{X}_{\mathrm{I} \times \mathrm{JKL}}=\mathbf{A}(\mathbf{D} \odot \mathbf{C} \odot \mathbf{B})^{\mathrm{T}}+\mathbf{E}_{\mathrm{I} \times \mathrm{JKL}}
$$

here,

$$
\begin{aligned}
\mathbf{X}_{\mathrm{I} \times \mathrm{JKL}}= & {\left[\mathbf{X}_{\ldots 11}, \mathbf{X}_{\ldots 21}, \ldots, \mathbf{X}_{\ldots \mathrm{K} 1}, \mathbf{X}_{\ldots 12}, \mathbf{X}_{\ldots 22}, \ldots, \mathbf{X}_{\ldots \mathrm{K} 2}, \ldots, \mathbf{X}_{\ldots \mathrm{KL}},\right.} \\
& \left.\mathbf{X}_{. . \mathrm{KL}}, \ldots, \mathbf{X}_{\ldots \mathrm{KL}}\right] \\
\mathbf{E}_{\mathrm{I} \times \mathrm{JKL}}= & {\left[\mathbf{E}_{.11}, \mathbf{E}_{.21}, \ldots, \mathbf{E}_{\ldots \mathrm{K} 1}, \mathbf{E}_{\ldots 12}, \mathbf{E}_{.22}, \ldots, \mathbf{E}_{\ldots \mathrm{K} 2}, \ldots, \mathbf{E}_{. \mathrm{KL}},\right.} \\
& \left.\mathbf{E}_{. \mathrm{KL}}, \ldots, \mathbf{E}_{\ldots \mathrm{KL}}\right], \\
\mathbf{X}_{\mathrm{J} \times \mathrm{KLI}}= & \mathbf{B}(\mathbf{A} \odot \mathbf{D} \odot \mathbf{C})^{\mathrm{T}}+\mathbf{E}_{\mathrm{J} \times \mathrm{KLI}}
\end{aligned}
$$

here,

$$
\begin{aligned}
\mathbf{X}_{\mathrm{J} \times \mathrm{KLI}}= & {\left[\mathbf{X}_{1 . .1}, \mathbf{X}_{1 . .2}, \ldots, \mathbf{X}_{1 . . \mathrm{L}}, \mathbf{X}_{2 . .1}, \mathbf{X}_{2 . .2}, \ldots, \mathbf{X}_{2 . . \mathrm{L}}, \ldots, \mathbf{X}_{\mathrm{I..1}}, \ldots,\right.} \\
& \left.\mathbf{X}_{\mathrm{I} . \mathrm{L}}\right] \\
\mathbf{E}_{\mathrm{J} \times \mathrm{KLI}}= & {\left[\mathbf{E}_{1 . .1}, \mathbf{E}_{1.2}, \ldots, \mathbf{E}_{1 . . \mathrm{L}}, \mathbf{E}_{2 . .1}, \mathbf{E}_{2 . .2}, \ldots, \mathbf{E}_{2 . . \mathrm{L}}, \ldots, \mathbf{E}_{\mathrm{II.1}}, \ldots,\right.} \\
& \left.\mathbf{E}_{\mathrm{I} . \mathrm{L}}\right], \\
\mathbf{X}_{\mathrm{K} \times \mathrm{LIJ}}= & \mathbf{C}(\mathbf{B} \odot \mathbf{A} \odot \mathbf{D})^{\mathrm{T}}+\mathbf{E}_{\mathrm{K} \times \mathrm{LIJ}}
\end{aligned}
$$

here,

$$
\begin{aligned}
& \mathbf{X}_{\mathrm{K} \times \mathrm{LIJ}}=\left[\mathbf{X}_{11 . .}, \mathbf{X}_{21 . .}, \ldots, \mathbf{X}_{11 . .}, \mathbf{X}_{12 . .}, \mathbf{X}_{22 . .}, \ldots, \mathbf{X}_{12 . .}, \ldots, \mathbf{X}_{1 \mathrm{~J} . .},\right. \\
& \left.\mathbf{X}_{2 \mathrm{~J} .}, \ldots, \mathbf{X}_{\mathrm{IJ} .}\right] \\
& \mathbf{E}_{\mathrm{K} \times \mathrm{LIJ}}=\left[\mathbf{E}_{11 . .}, \mathbf{E}_{21 . .}, \ldots, \mathbf{E}_{11 . .}, \mathbf{E}_{12 . .}, \mathbf{E}_{22 . .}, \ldots, \mathbf{E}_{12 . .}, \ldots, \mathbf{E}_{1 \mathrm{~J} . .},\right. \\
& \left.\mathbf{E}_{2 \mathrm{~J}}, \ldots, \mathbf{E}_{\mathrm{IJ.}}\right] \text {, } \\
& \mathbf{X}_{\mathrm{L} \times \mathrm{IJK}}=\mathbf{D}(\mathbf{C} \odot \mathbf{B} \odot \mathbf{A})^{\mathrm{T}}+\mathbf{E}_{\mathrm{L} \times \mathrm{IJK}}
\end{aligned}
$$

here,

$$
\begin{aligned}
& \mathbf{X}_{\mathrm{LXIJK}}=\left[\mathbf{X}_{.11}, \mathbf{X}_{.21,}, \ldots, \mathbf{X}_{\mathrm{JI}}, \mathbf{X}_{.12}, \mathbf{X}_{.22}, \ldots, \mathbf{X}_{\mathrm{J} 2,}, \ldots, \mathbf{X}_{.1 \mathrm{~K}}\right. \\
& \left.\mathbf{X}_{\text {.2K. }}, \ldots, \mathbf{X}_{\text {.J. }}\right] \\
& \mathbf{E}_{\mathrm{L} \times \mathrm{IJK}}=\left[\mathbf{E}_{.11}, \mathbf{E}_{221}, \ldots, \mathbf{E}_{. J 1}, \mathbf{E}_{.12}, \mathbf{E}_{.22}, \ldots, \mathbf{E}_{\mathrm{J} 2}, \ldots, \mathbf{E}_{.1 \mathrm{~K} .},\right. \\
& \mathbf{E}_{.2 \mathrm{~K} .}, \ldots, \mathbf{E}_{\mathrm{JK}} \text {. }
\end{aligned}
$$


PARAFAC-ALS ${ }^{8,33}$ is one of the classical trilinear decomposition algorithms. As an extension of PARAFAC-ALS to quadrilinear, a four-way PARAFAC algorithm is based on the alternating least-squares principle for minimizing four direct loss functions of the quadrilinear model in a stretched matrix form. These loss functions of four-way PARAFAC are as follows:

$$
\begin{aligned}
& \sigma(\mathbf{A})=\left\|\mathbf{X}_{\mathrm{I} \times \mathrm{JKL}}-\mathbf{A}(\mathbf{C} \odot \mathbf{B} \odot \mathbf{D})^{\mathrm{T}}\right\|_{\mathrm{F}}^{2}, \\
& \sigma(\mathbf{B})=\left\|\mathbf{X}_{\mathrm{J} \times \mathrm{KLI}}-\mathbf{B}(\mathbf{A} \odot \mathbf{D} \odot \mathbf{C})^{\mathrm{T}}\right\|_{\mathrm{F}}^{2}, \\
& \sigma(\mathbf{C})=\left\|\mathbf{X}_{\mathrm{K} \times \mathrm{LIJ}}-\mathbf{C}(\mathbf{B} \odot \mathbf{A} \odot \mathbf{D})^{\mathrm{T}}\right\|_{\mathrm{F}}^{2}, \\
& \sigma(\mathbf{D})=\left\|\mathbf{X}_{\mathrm{L} \times \mathrm{IJK}}-\mathbf{D}(\mathbf{C} \odot \mathbf{B} \odot \mathbf{A})^{\mathrm{T}}\right\|_{\mathrm{F}}^{2} .
\end{aligned}
$$

On the basis of the above-mentioned loss functions, the four-way PARAFAC method processes by the alternating least-squares minimization scheme according to Eqs. (6) - (9), such as finding a solution of $\mathbf{A}$ for fixed $\mathbf{B}, \mathbf{C}$ and $\mathbf{D}$. In the same way, $\mathbf{B}, \mathbf{C}, \mathbf{D}$ can be computed. The four matrices are updated through using the strict least-squares principle as follows:

$$
\begin{aligned}
& \mathbf{A}=\mathbf{X}_{\mathrm{I} \times \mathrm{JKL}}\left[(\mathbf{D} \odot \mathbf{C} \odot \mathbf{B})^{\mathrm{T}}\right]^{+}, \\
& \mathbf{B}=\mathbf{X}_{\mathrm{J} \times \mathrm{KLI}}\left[(\mathbf{A} \odot \mathbf{D} \odot \mathbf{C})^{\mathrm{T}}\right]^{+}, \\
& \mathbf{C}=\mathbf{X}_{\mathrm{K} \times \mathrm{LIJ}}\left[(\mathbf{B} \odot \mathbf{A} \odot \mathbf{D})^{\mathrm{T}}\right]^{+}, \\
& \mathbf{D}=\mathbf{X}_{\mathrm{L} \times \mathrm{IJK}}\left[(\mathbf{C} \odot \mathbf{B} \odot \mathbf{A})^{\mathrm{T}}\right]^{+} .
\end{aligned}
$$

A typical iterative procedure for four-way PARAFAC can be carried out as follows:

1. Estimate the number of factors, $N$;

2. Initialize $\mathbf{A}, \mathbf{B}$, and $\mathbf{C}$, randomly;

3. Compute D using Eq. (13);

4. Compute A, B, C using Eqs. (10), (11), (12) and scale A, B,

C to be columnwisely normalized, respectively;

5. Compute D using Eq. (13);

6. Repeat steps 4 and 5 until a stopping criterion is satisfied. In the iterative process, the stopping criterion is:

$$
\left|\frac{\mathrm{SSR}^{(\mathrm{m})}-\mathrm{SSR}^{(\mathrm{m}-1)}}{\mathrm{SSR}^{(\mathrm{m}-1)}}\right|<\varepsilon
$$

where $\varepsilon$ is generally set to be $10^{-6}, \mathrm{SSR}=\|\underline{\mathbf{E q}}\|_{\mathrm{F}}^{2}, m$ is the iteration number and $\left\|\underline{\mathbf{E}}_{\mathrm{q}}\right\|_{\mathrm{F}}=\left(\sum_{\mathrm{i}}^{\mathrm{I}} \sum_{\mathrm{j}}^{\mathrm{J}} \sum_{\mathrm{k}}^{\mathrm{K}} \sum_{1}^{\mathrm{L}} e_{\mathrm{ijk} \mathrm{l}}^{2}\right)^{1 / 2}$. A maximal number of 3000 iterations are set to avoid unduly slow convergence.

In this way, $\mathbf{D}$ and these normalized $\mathbf{A}, \mathbf{B}, \mathbf{C}$ can be acquired. After calibration is done with $\mathbf{A}, \mathbf{B}, \mathbf{C}, \mathbf{D}$, the concentrations of the analyte of interest in predicted samples as well as their profiles related to $\mathbf{A}, \mathbf{B}, \mathbf{C}$ can be obtained.

\section{Four-way SWANRF algorithm}

Four-way SWANRF can be regarded as an extension of SWANRF, whose formulation is described in detail in the literature, reported by Nie et al. ${ }^{12}$ For a quadrilinear model, one can obtain the following new residues:

$$
\begin{aligned}
& \mathbf{X}_{\mathrm{I} \times \mathrm{JKL}} \mathbf{P}^{+}=\mathbf{A P P}^{+}+\mathbf{E}_{\mathrm{I} \times \mathrm{JKL}} \mathbf{P}^{+}, \text {here, } \mathbf{P}=(\mathbf{D} \odot \mathbf{C} \odot \mathbf{B})^{\mathrm{T}}, \\
& \mathbf{X}_{\mathrm{J} \times \mathrm{KLI}} \mathbf{Q}^{+}=\mathbf{B} \mathbf{Q} \mathbf{Q}^{+}+\mathbf{E}_{\mathrm{J} \times \mathrm{KLI}} \mathbf{Q}^{+}, \text {here, } \mathbf{Q}=(\mathbf{A} \odot \mathbf{D} \odot \mathbf{C})^{\mathrm{T}},
\end{aligned}
$$

$$
\begin{aligned}
& \mathbf{X}_{\mathrm{K} \times \mathrm{LIJ}} \mathbf{R}^{+}=\mathbf{C R R}^{+}+\mathbf{E}_{\mathrm{K} \times \mathrm{LIJ}} \mathbf{R}^{+}, \text {here, } \mathbf{R}=(\mathbf{B} \odot \mathbf{A} \odot \mathbf{D})^{\mathrm{T}}, \\
& \mathbf{X}_{\mathrm{L} \times \mathrm{IJK}} \mathbf{S}^{+}=\mathbf{D S S}^{+}+\mathbf{E}_{\mathrm{L} \times \mathrm{IJK}} \mathbf{S}^{+}, \text {here, } \mathbf{S}=(\mathbf{C} \odot \mathbf{B} \odot \mathbf{A})^{\mathrm{T}} .
\end{aligned}
$$

Four-way SWANRF makes use of these four new residues to construct four objective functions, respectively. One could solve the quadrilinear model based on truncated least squares by alternatively optimizing the following four objective functions:

$$
\begin{aligned}
& \mathrm{S}(\mathbf{A})=\left\|\left(\mathbf{X}_{\mathrm{I} \times \mathrm{JKL}} \mathbf{P}^{+}-\mathbf{A} \mathbf{P} \mathbf{P}^{+}\right) \times \mathbf{W}_{\mathrm{A}}\right\|_{\mathrm{F}}^{2}, \\
& \text { here, } \mathbf{W}_{\mathrm{A}}=\operatorname{diag}\left(\operatorname{sqrt}\left(\mathbf{1} . \operatorname{diagm}\left(\mathbf{A A}^{\mathrm{T}}\right)\right)\right) \text {, } \\
& \mathrm{S}(\mathbf{B})=\left\|\left(\mathbf{X}_{\mathrm{J} \times \mathrm{KLI}} \mathbf{Q}^{+}-\mathbf{B} \mathbf{Q} \mathbf{Q}^{+}\right) \times \mathbf{W}_{\mathrm{B}}\right\|_{\mathrm{F}}^{2}, \\
& \text { here, } \mathbf{W}_{\mathrm{B}}=\operatorname{diag}\left(\operatorname{sqrt}\left(\mathbf{1} . \operatorname{diagm}\left(\mathbf{B B}^{\mathrm{T}}\right)\right)\right) \text {, } \\
& \mathrm{S}(\mathbf{C})=\left\|\left(\mathbf{X}_{\mathrm{K} \times \mathrm{LIJ}} \mathbf{R}^{+}-\mathbf{C R R}^{+}\right) \times \mathbf{W}_{\mathrm{C}}\right\|_{\mathrm{F}}^{2}, \\
& \text { here, } \mathbf{W}_{\mathrm{C}}=\operatorname{diag}\left(\operatorname{sqrt}\left(\mathbf{1} . / \operatorname{diagm}\left(\mathbf{C C}^{\mathrm{T}}\right)\right)\right) \text {, } \\
& \mathbf{S}(\mathbf{D})=\left\|\left(\mathbf{X}_{\mathrm{L} \times \mathrm{IJK}} \mathbf{S}^{+}-\mathbf{D S S}^{+}\right) \times \mathbf{W}_{\mathrm{D}}\right\|_{\mathrm{F}}^{2}, \\
& \text { here, } \mathbf{W}_{\mathrm{D}}=\operatorname{diag}\left(\operatorname{sqrt}\left(\mathbf{1} . / \operatorname{diagm}\left(\mathbf{D D}^{\mathrm{T}}\right)\right)\right) \text {. }
\end{aligned}
$$

Here, $\mathbf{W}_{\mathrm{A}}, \mathbf{W}_{\mathrm{B}}, \mathbf{W}_{\mathrm{C}}$ and $\mathbf{W}_{\mathrm{D}}$ are weight factors. The weight factor is accompanying outcome by operating Moore-Penrose generalized inverse on the stretched matrices. At this point, the new weight factors are different from the original SWANRF that it makes linearity information projection to the space, which is expand from $\mathbf{A} \mathbf{A}^{\mathrm{T}}, \mathbf{B} \mathbf{B}^{\mathrm{T}}, \mathbf{C C}^{\mathrm{T}}$ and $\mathbf{D D} \mathbf{D}^{\mathrm{T}}$. It was created with the purpose of getting rid of nonlinearity information and achieving a stable result for the condition of high noise. There is a figure for comparing the minimization process with and without a weight factor in Supporting Information. According to the above-mentioned objective functions, one minimizes those in an alternating manner; that is, $\mathbf{D}$ minimizes $\mathbf{S}(\mathbf{D})$ (Eq. (21)) for fixed A, B, and $\mathbf{C}$; $\mathbf{A}$ minimizes $\mathbf{S}(\mathbf{A})($ Eq. (18)) for fixed $\mathbf{B}, \mathbf{C}$, and $\mathbf{D} ; \mathbf{B}$ minimizes $\mathbf{S}(\mathbf{B})$ (Eq. (19)) for fixed $\mathbf{A}, \mathbf{C}$, and $\mathbf{D} ; \mathbf{C}$ minimizes $\mathbf{S}(\mathbf{C})$ (Eq. (20)) for fixed $\mathbf{A}, \mathbf{B}$, and D. Thus, A, B, C and $\mathbf{D}$ can be calculated iteratively as follows:

$$
\begin{aligned}
& \mathbf{A}=\left(\mathbf{X}_{\mathrm{I} \times \mathrm{JKL}} \mathbf{P}^{+} \mathbf{W}_{\mathrm{A}} \mathbf{W}_{\mathrm{A}}\left(\mathbf{P}^{\mathrm{T}}\right)^{+} \mathbf{P}^{\mathrm{T}}\right)\left(\mathbf{P} \mathbf{P}^{+} \mathbf{W}_{\mathrm{A}} \mathbf{W}_{\mathrm{A}}\left(\mathbf{P}^{\mathrm{T}}\right)^{+} \mathbf{P}^{\mathrm{T}}\right)^{+}, \\
& \mathbf{B}=\left(\mathbf{X}_{\mathrm{J} \times \mathrm{KLI}} \mathbf{Q}^{+} \mathbf{W}_{\mathrm{B}} \mathbf{W}_{\mathrm{B}}\left(\mathbf{Q}^{\mathrm{T}}\right)^{+} \mathbf{Q}^{\mathrm{T}}\right)\left(\mathbf{Q} \mathbf{Q}^{+} \mathbf{W}_{\mathrm{B}} \mathbf{W}_{\mathrm{B}}\left(\mathbf{Q}^{\mathrm{T}}\right)^{+} \mathbf{Q}^{\mathrm{T}}\right)^{+}, \\
& \mathbf{C}=\left(\mathbf{X}_{\mathrm{K} \times \mathrm{LJ} \mathrm{R}} \mathbf{R}^{+} \mathbf{W}_{\mathrm{C}} \mathbf{W}_{\mathrm{C}}\left(\mathbf{R}^{\mathrm{T}}\right)^{+} \mathbf{R}^{\mathrm{T}}\right)\left(\mathbf{R} \mathbf{R}^{+} \mathbf{W}_{\mathrm{C}} \mathbf{W}_{\mathrm{C}}\left(\mathbf{R}^{\mathrm{T}}\right)^{+} \mathbf{R}^{\mathrm{T}}\right)^{+}, \\
& \mathbf{D}=\left(\mathbf{X}_{\mathrm{L} \times \mathrm{IJK}} \mathbf{S}^{+} \mathbf{W}_{\mathrm{D}} \mathbf{W}_{\mathrm{D}}\left(\mathbf{S}^{\mathrm{T}}\right)^{+} \mathbf{S}^{\mathrm{T}}\right)\left(\mathbf{S} \mathbf{S}^{+} \mathbf{W}_{\mathrm{D}} \mathbf{W}_{\mathrm{D}}\left(\mathbf{S}^{\mathrm{T}}\right)^{+} \mathbf{S}^{\mathrm{T}}\right)^{+} .
\end{aligned}
$$

An iterative procedure for four-way SWANRF is similar to the above one for the four-way PARAFAC.

Obviously, four-way PARAFAC provides strict least-squares solutions in a mathematical sense, whose loss function is a monotonic decline and spectral profiles smooth. However, in each iterative procedure, more or less deviations between the loading matrices $(\mathbf{A}, \mathbf{B}, \mathbf{C})$ and the score matrix $(\mathbf{D})$ and their corresponding underlying matrices exist. These deviations which will be retained and transferred through an iterative process, leading to the slow convergence, perturbations to four-way PARAFAC and even making the four-way PARAFAC drop into a "swamp", especially encountering serious collinearity. Furthermore, four-way PARAFAC leaves some trashy error in the three loadings, or losses some valid information to the residue in order to ensure the sum of squares of the residue minimum.

Theoretically, the convergence rate and stability of the four-way SWANRF algorithm would be improved through shuffling the computational matrices. The redistribution of 
residue will increase the ability to grasp valid information. In the meantime, four-way SWANRF can conquer the collinear problem to some extent by introducing weight terms.

\section{Simulated and Experimental}

\section{Simulated $L C \times L C-D A D$ data}

A four-way data array generated by an LC $\times$ LC-DAD on twelve samples with four species was simulated. A four-way data array $(30 \times 50 \times 100 \times 12)$ was constructed in the way provided in Supporting Information. The accuracy of the four-way computational results can be judged by the consistency value between resolved and actual profiles. A value of COS less than 0.99 means malfunction. ${ }^{49}$

\section{Apparatus}

All of the fluorometric measurements were achieved on a F-4500 fluorescence spectrophotometer (HITACHI) equipped with a Xenon lamp and interfaced to a personal computer. In all cases, a $1.00 \mathrm{~cm}$ quartz cell was used. All calculations were implemented in the Matlab environment on a personal computer under the Windows 7 operating system.

\section{Parameters}

The excitation wavelengths were set from 200.0 to $300.0 \mathrm{~nm}$ with an interval of $2.0 \mathrm{~nm}$, and the emission wavelength varied from 309.0 to $414.0 \mathrm{~nm}$ with an interval of $3.0 \mathrm{~nm}$. The slit width was $5.0 / 5.0 \mathrm{~nm}$. The scan rate was $12000 \mathrm{~nm} \mathrm{~min}^{-1}$.

\section{Reagents}

All solvents used were of analytical grade. 5-Hydroxytryptamine hydrochloride was obtained from the National Institute for the Control of Pharmaceutical and Biological Products (NICPBP). The water used was doubly distilled. The plasma was purchased from the Blood Center in Changsha.

\section{Sample preparation}

The stock solutions of serotonin were prepared by dissolving $10.1 \mathrm{mg}$ of reagent (Acros Organics) in $100.00 \mathrm{ml}$ of doubly distilled water. The serotonin diluent was obtained by diluting the stock solution with doubly distilled water with a concentration of $11.87 \mathrm{pmol} \mathrm{ml}^{-1}$. The plasma diluent was obtained from diluting the plasma $(1.00 \mathrm{~mL})$ with doubly distilled water $(20.00 \mathrm{~mL})$. Appropriate serotonin solutions of different concentrations were prepared by diluting in a borate buffer solution. And each concentration of serotonin solutions was prepared in four $\mathrm{pH}$ values $(9.10,9.22,9.40$, and 9.50).

\section{Data array}

Thus a four-way data array of an excitation-emission-pH-sample $(51 \times 36 \times 4 \times 15)$ was thereby assembled. For each $\mathrm{pH}$, five calibration samples, as well as five plasmatic and five spiked plasmatic samples were prepared. The serotonin concentrations of the calibration samples were in the range of $0.237-1.187 \mathrm{pmol} \mathrm{mL}^{-1}$. Both of the linear regression coeffficients $\left(R^{2}\right)$, which result from two algorithms, were 0.9980. Besides, the concentrations of serotonin and the volume of plasma diluent added in plasmatic and spiked plasmatic samples are given in Tables 2 and 3.
Table 2 Content of serotonin in plasma resolved by four-way SWANRF and four-way PARAFAC

\begin{tabular}{|c|c|c|c|}
\hline \multirow{2}{*}{\multicolumn{2}{|c|}{ Plasma/ $\mu \mathrm{L}$}} & \multicolumn{2}{|c|}{ Serotonin content $/ \mathrm{nmol} \mathrm{mL}^{-1}$} \\
\hline & & Four-way SWANRF & Four-way PARAFAC \\
\hline P1 & 300 & 0.320 & 0.344 \\
\hline P2 & 300 & 0.336 & 0.360 \\
\hline P3 & 500 & 0.315 & 0.338 \\
\hline P4 & 500 & 0.325 & 0.348 \\
\hline P5 & 500 & 0.325 & 0.348 \\
\hline \multicolumn{2}{|c|}{$\begin{array}{l}\text { Average content/ } \\
\text { nmol mL }\end{array}$} & $0.324 \pm 0.005$ & $0.348 \pm 0.006$ \\
\hline \multicolumn{2}{|c|}{ T-test } & \multicolumn{2}{|c|}{6.67} \\
\hline
\end{tabular}

T-test $=\left(\bar{x}_{1}-\bar{x}_{2}\right) /(s / \sqrt{n})$, where $\bar{x}$ is average content, $n$, degree of freedom and confidence level $99.8 \%, t_{0.002}^{4}=7.17$.

Table 3 Recovery of serotonin in spiked plasmatic samples resolved by four-way SWANRF and four-way PARAFAC

\begin{tabular}{|c|c|c|c|c|}
\hline & \multirow{2}{*}{ 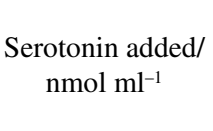 } & \multirow[b]{2}{*}{ Plasma/ $/ \mu 1$} & \multicolumn{2}{|c|}{ Recovery, \% } \\
\hline & & & $\begin{array}{l}\text { Four-way } \\
\text { SWANRF }\end{array}$ & $\begin{array}{l}\text { Four-way } \\
\text { PARAFAC }\end{array}$ \\
\hline S1 & 0.324 & 200 & 102.5 & 102.5 \\
\hline S2 & 0.324 & 200 & 102.9 & 102.8 \\
\hline S3 & 0.324 & 200 & 105.9 & 105.9 \\
\hline S4 & 0.648 & 400 & 76.8 & 76.3 \\
\hline S5 & 0.648 & 400 & 85.5 & 85.3 \\
\hline \multicolumn{3}{|c|}{ Average recovery ,\% } & $\begin{array}{c}94.7 \pm 10.8 \\
1.09\end{array}$ & $\begin{array}{c}94.6 \pm 11.0 \\
1.11\end{array}$ \\
\hline
\end{tabular}

T-test $=\left(\bar{x}-\mu_{0}\right) /(s / \sqrt{n})$, where $\bar{x}$ is the average recovery, $\mu_{0}, 100 \%$, $n$, degree of freedom and confidence level $99.8 \%, t_{0.002}^{4}=7.17$.

\section{Results and Discussion}

\section{Simulated data arrays}

For the sake of comparison, a three-way data set, which was constructed by leaving out the second dimensionality of the simulated four-way data set, was calculated by the PARAFAC and SWANRF algorithms (10 times). From Table S1 (Supporting Information), all of the COSs were less than 0.99, indicating that the heavy collinearity data can not be resolved by a three-way algorithm like PARAFAC and SWANRF. Fortunately, after the fourth mode was brought in, the results for 10 times were very satisfactory. Table S2 (Supporting Information) has shown the COS that can reveal results calculated by two four-way algorithms. We can see that, the four-way PARAFAC made a mistake once, while the four-way SWANRF acted well each time. In Fig. 2, the solid lines mean the stimulated profiles, while dashed lines stand for resolved profiles. The first six figures are three-way data stimulating and resolved results. With regard to the four components, $\mathbf{A}$ is the chromatogram, $\mathbf{B}$ represents UV the spectrum and $\mathbf{C}$ means the relative concentrate spectrum. It shows that the resolved profiles (dashed lines) do not overlap with the actual ones (solid lines). This means that the results treated by two three-way algorithms were not satisfactory owing to the heavy collinearity of simulated data. In the meantime, the last eight profiles are four-way data stimulating and resolved results. Accordingly, with regard to the four components, $\mathbf{A}$ and $\mathbf{B}$ are chromatograms, C represents UV spectra and D means relative concentration 

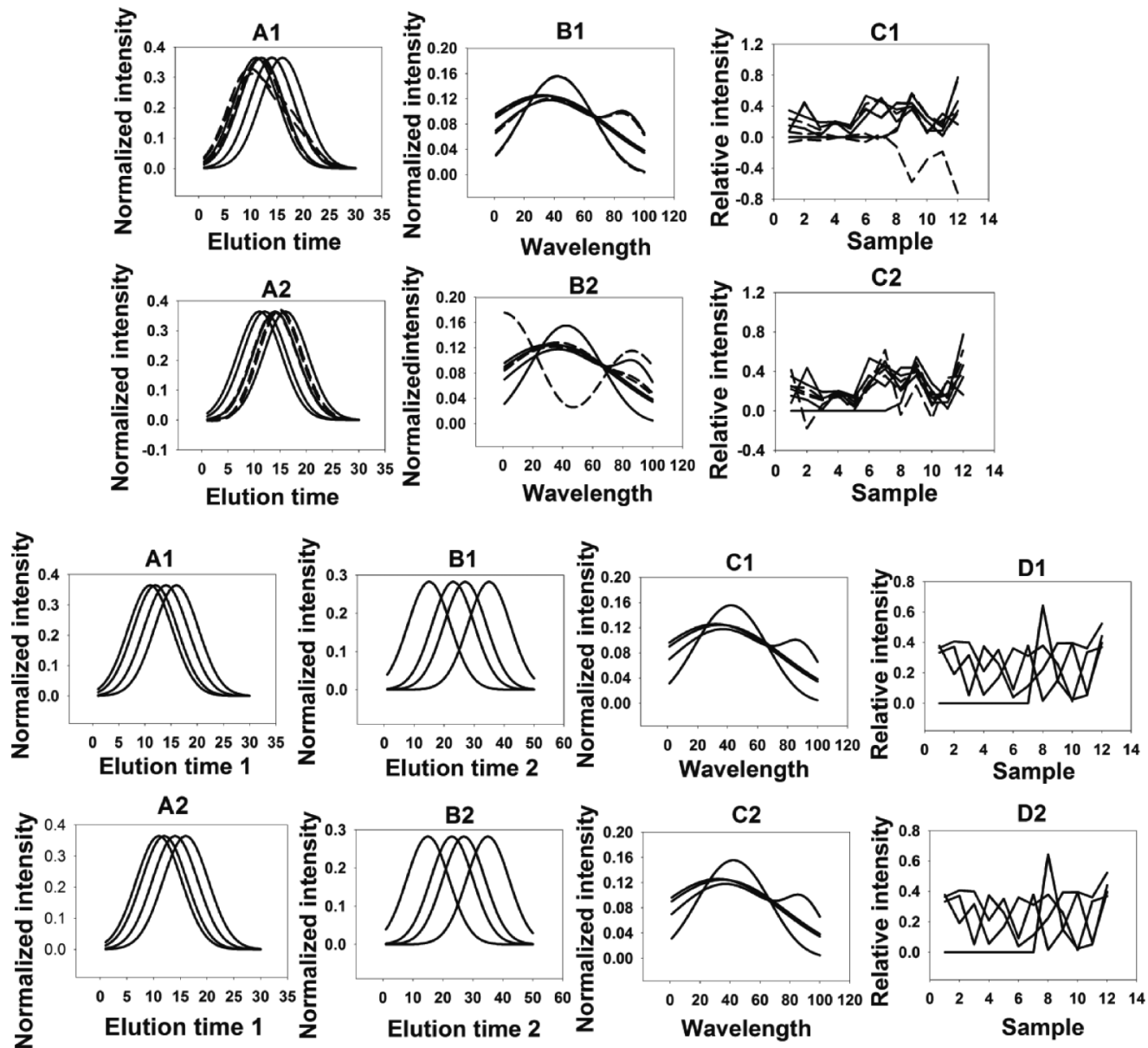

Fig. 2 Resolved and true profiles of simulated three-way and four-way data. The solid lines mean the simulated profiles, and dashed lines represent the resolved profiles.

profiles. It illustrates that the resolved profiles (dashed lines) overlap with the actual ones (solid lines). The profiles resulting from four-way algorithms show great resemblance to the actual ones. Hence, we come to the conclusion that the introduction of a fourth mode is competent to relieve the serious problem of collinearity, which three-way algorithms could not handle. We think that the advantage belongs to a "third-order advantage". Besides, the new four-way SWANRF algorithm seems to be a promising one, which can be used for decomposition of fourway data.

\section{Application to analyze serotonin in plasma samples}

In this section, third-order calibration is brought into determining serotonin in plasma samples. Figure 3 shows a typical series of EEMs for serotonin standards $\left(1.187 \mathrm{pmol} \mathrm{mL}^{-1}\right)$, taken at different $\mathrm{pH}$ values, ranging from 9.10 to 9.50 , indicating that the fluorescence intensity of serotonin decreases as the $\mathrm{pH}$ increases.

The four-way data array of the excitation-emission-pH-sample $(51 \times 36 \times 4 \times 15)$ was constructed with calibration samples, plasma samples plus spiked plasma samples at four $\mathrm{pH}$ levels. The data array was analyzed by four-way SWANRF and PARAFAC algorithms. Core consistency analysis required the consideration of two fluorophores, when the set of sixty samples was analyzed by the two algorithms, as expected from the sample composition. Meanwhile, other component numbers would lead to poorly defined profiles for the additional constituents.

As a result, the estimated and actual relative excitation, emission, $\mathrm{pH}$ profiles, and relative absorbance intensity of different samples are shown in Fig. 4. As demonstrated in Fig. 4, excitation and emission profiles of serotonin are similar to those of background from the plasma. It is out of the question to conduct quantitative analysis without "physical or chemical separation". Also, it is hard to take advantage of "second-order calibration" for the high colinearity of excitation and emission profiles, not to mention that the fluorescence intensity of the background is much stronger than that of the analyte. Fortunately, we can turn to third-order calibration for help. It can be appreciated that the fluorescence intensity of the analyte decreases considerably as a function of the $\mathrm{pH}$ value, while the fluorescence intensity of the background is not that sensitive to the change of $\mathrm{pH}$. Thus, the third-order calibration can deal with this four-way data array. The profiles resolved by the above-mentioned four-way algorithms match quite well with the true signal, which was normalized. Based on the concentration of calibration samples, it is easy to compute the content of serotonin in plasma, and the recoveries of spiked plasma samples. The content of serotonin and the recoveries of spiked plasma samples are summarized in Tables 2 and 3. The contents of serotonin in plasma are reasonable as references. ${ }^{41-46}$

Sensitivity (SEN) is a figure that characterizes the calibration model, and tells to what extent the response due to a particular analyte varies as a function of its concentration. The sensitivity 

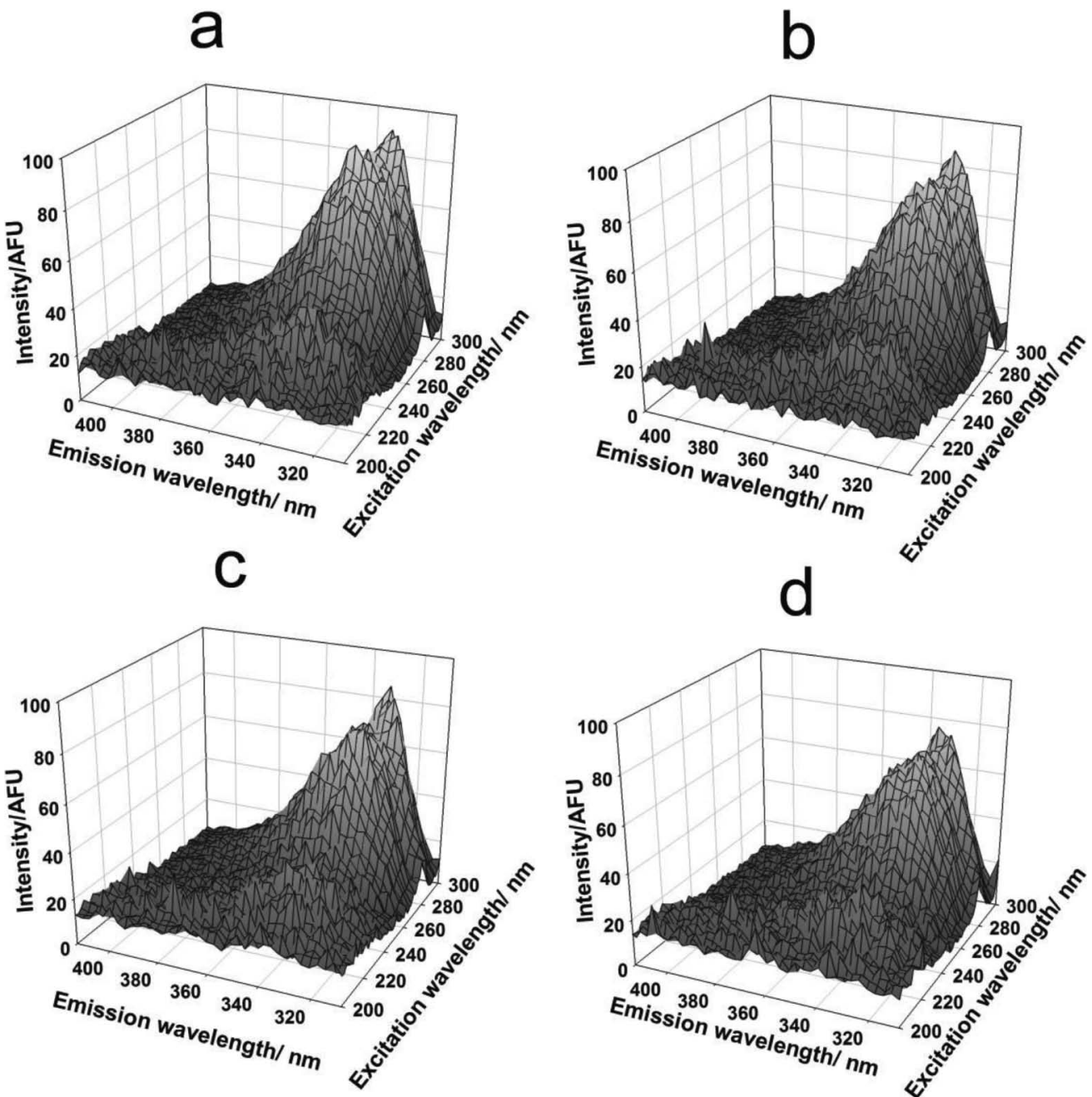

Fig. 3 Three-dimensional plots of the excitation-emission matrix fluorescence spectra for serotonin of a series of $\mathrm{pH}$. The $\mathrm{pH}$ values selected for measurement are: a (9.10), b (9.22), c (9.40), and d (9.50), respectively.

in the context of univariate calibration is defined as the slope of the calibration curve. It is essentially a differential of the response with regard to the concentration. A reliable method that is efficient to be used to calculate SEN in four-way data array was chosen in this paper based on literature reported by Olivieri and Faber. ${ }^{50}$ SEN was calculated as: $\mathrm{SEN}=s_{\mathrm{n}} \| n$th row of $\left(\mathbf{P}_{\text {zunx }} \mathbf{Z}_{\text {cal }}\right)^{+} \|^{-1}, \mathbf{P}_{\text {zunx }}=\mathbf{I}-\mathbf{Z}_{\text {unx }} \mathbf{Z}_{\text {unx }}{ }^{+}$. $\mathbf{Z}_{\text {unx }}$ is, corresponding, solely due to the profiles for the unexpected constituents, while $\mathbf{Z}_{\mathrm{cal}}$ corresponds to the profiles for the calibrated analytes. Notice that $s_{\mathrm{n}}$ in the above was measured from scores versus concentrations, which is regularly employed from four-way algorithm analyte prediction. The values of SEN, which resulted from four-way SWANRF and four-way PARAFAC, are 7783.9

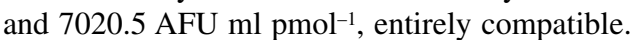

\section{Conclusion}

As has been stated, a novel algorithm, the four-way SWANRF, was developed for the quadrilinear analysis of four-way data arrays and third-order calibration. As well as four-way PARAFAC, it can be employed to explore the analytical characteristics of higher-order data, and it is further indicated that the introduction of the fourth mode can relieve the serious problem of collinearity, which three-way algorithms can not easily to treat. By treating a simulated data array, the presented results have shown that the four-way SWANRF algorithm can obtain stable results when high level of colinearity exists. Furthermore, the quantitative analysis of serotonin in human plasma by the third-order calibration method seems to be both efficient and sensitive.

\section{Acknowledgements}

The authors would like to acknowledge financial support by the National Natural Science Foundation of China (Grant Nos. 21175041 and 20775025) and the National Basic Research Program (No. 2012CB910602).

\section{Supporting Information}

The Supporting Information is about the figure for comparing the minimization process with and without a weight factor, the simulated LC $\times$ LC-DAD data and the value of COS, which is 

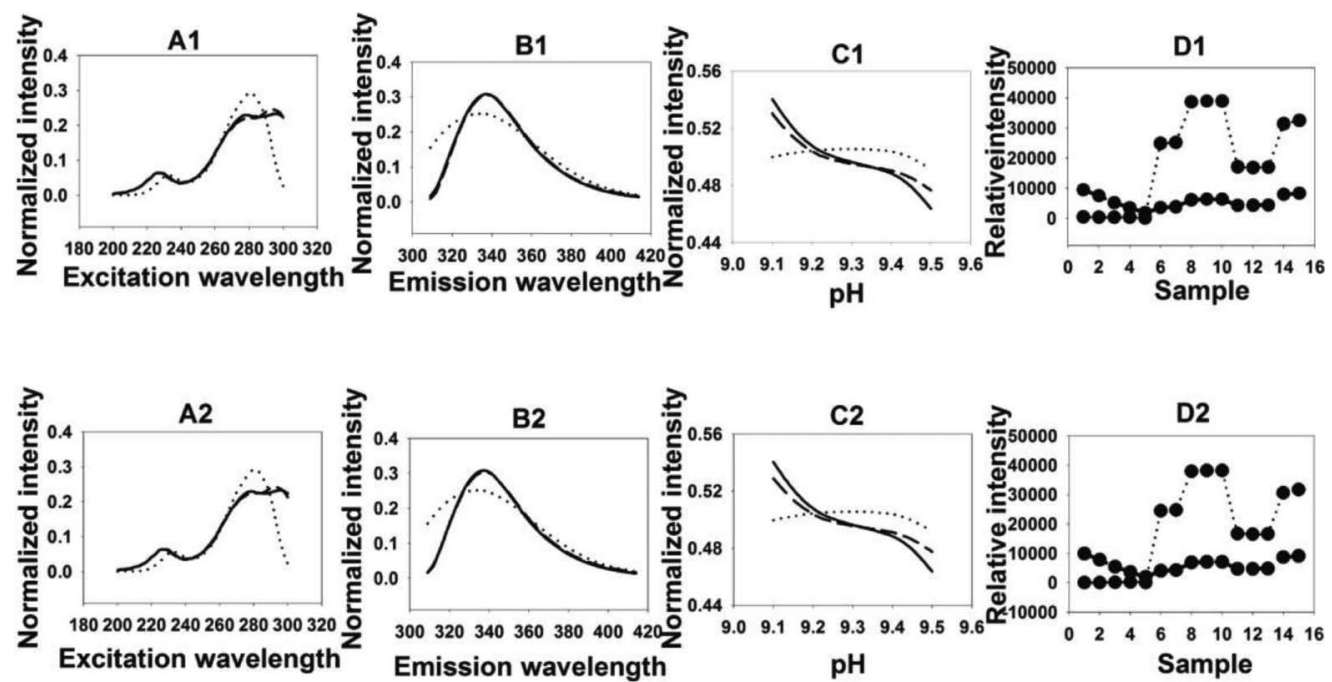

Fig. 4 Resolved and actual excitation, emission, $\mathrm{pH}$ spectra and the relative absorbance intensity of different samples. The solid and medium dash lines mean the actual and resolved profiles, respectively. The dashed lines stand for the profiles of background.

calculated by three-way and four-way algorithms (10 times). This material is available free of charge on the Web at http:// www.jsac.or.jp/analsci/.

\section{References}

1. K. S. Booksh and B. R. Kowalski, Anal. Chem., 1994, 66, 782A.

2. Y. Wang, O. S. Borgen, B. R. Kowalski, M. Gu, and F. Turecek, J. Chemom., 1993, 7, 117.

3. E. Sanchez and B. R. Kowalski, J. Chemom., 1988, 2, 265.

4. E. Sanchez and B. R. Kowalski, Anal. Chem., 1986, 58, 496.

5. B. E. Wilson, E. Sanchez, and B. R. Kowalski, J. Chemom., 1989, 3, 493.

6. K. Faber, A. Lorber, and B.R. Kowalski, J. Chemom., 1997, $11,95$.

7. J. D. Carroll and J. J. Chang, Psychometrika, 1970, 35, 283.

8. R. Bro, Chemom. Intell. Lab. Syst., 1997, 38, 149.

9. R. A. Harshman and M. E. Lundy, Comput. Stat. Data An., 1994, 18, 39.

10. R. A. Harshman, UCLA Working Papers in Phonetics., 1970, 16,1 .

11. H. L. Wu, M. Shibukawa, and K. Oguma, J. Chemom., 1998, 12,1 .

12. J. F. Nie, H. L. Wu, S. R. Zhang, Y. J. Yu, and R. Q. Yu, Anal. Methods, 2010, 2, 1918.

13. Z. P. Chen, H. L. Wu, J. H. Jiang, Y. Li, and R. Q. Yu, Chemom. Intell. Lab. Syst., 2000, 52, 75.

14. A. L. Xia, H. L. Wu, D. M. Fang, Y. J. Ding, L. Q. Hu, and R. Q. Yu, J. Chemom., 2005, 19, 65.

15. H. L. Wu, J. F. Nie, Y. J. Yu, and R. Q. Yu, Anal. Chim. Acta, 2009, 650, 131.

16. M. Esteban, C. Ariño, J. Díaz-Cruz, M. Díaz-Cruz, and R. Tauler, TrAC, Trends Anal. Chem., 2000, 19, 49.

17. M. Linder and R. Sundberg, Chemom. Intell. Lab. Syst., 1998, 42, 159.

18. M. Linder and R. Sundberg, J. Chemom., 2002, 16, 12.

19. J. Hur and J. Cho, Sensors, 2012, 12, 972.
20. T. Pagano, A. D. Ross, J. Chiarelli, and J. E. Kenny, J. Environ. Monit., 2012, 14, 937

21. L. M. Zou, J. H. Li, D. G. Jiang, and Y. L. Wang, Advanced Materials Res., 2012, 356, 2536.

22. J. Wu, H. Zhang, L. M. Shao, and P. J. He, Environ. Pollut., 2012, 162, 63.

23. J. V. Simpson, M. Burke, and R. D. Jiji, J. Chemom., 2011, 25, 101.

24. J. F. Nie, H. L. Wu, A. L. Xia, S. H. Zhu, Y. C. Bian, S. F. Li, and R. Q. Yu, Anal. Sci., 2007, 23, 1377.

25. A. Pasamontes and M. Callao, Anal. Chim. Acta, 2004, $515,159$.

26. A. L. Xia, H. L. Wu, S. F. Li, S. H. Zhu, Y. Zhang, Q. J. Han, and R. Q. Yu, Talanta, 2007, 73, 606.

27. E. Peré-Trepat, A. Hildebrandt, D. Barceló, S. Lacorte, and R. Tauler, Chemom. Intell. Lab. Syst., 2004, 74, 293.

28. A. Olivieri, G. Escandar, and A. Munoz de la Pena, TrAC, Trends Anal. Chem., 2011, 30, 607.

29. G. M. Escandar, P. C. Damiani, H. C. Goicoechea, and A. C. Olivieri, Microchem. J., 2006, 82, 29.

30. M. L. Nahorniak, G. A. Cooper, Y. C. Kim, and K. S. Booksh, Analyst, 2004, 130, 85.

31. A. C. Olivieri, J. A. Arancibia, A. M. de la Peña, I. DuranMeras, and A. E. Mansilla, Anal. Chem., 2004, 76, 5657.

32. J. A. Arancibia, A. C. Olivieri, D. B. Gil, A. E. Mansilla, I. Duran-Meras, and A. M. de la Pena, Chemom. Intell. Lab. Syst., 2006, 80, 77.

33. A. L. Xia, H. L. Wu, S. F. Li, S. H. Zhu, L. Q. Hu, and R. Q. Yu, J. Chemom., 2007, 21, 133.

34. H. Y. Fu, H. L. Wu, Y. J. Yu, L. L. Yu, S. R. Zhang, J. F. Nie, S. F. Li, and R. Q. Yu, J. Chemom., 2011, 25, 408.

35. M. M. Rapport, A. A. Green, and I. H. Page, J. Biol. Chem., 1948, 176, 1243.

36. A. Sirek and O. Sirek, Can. Med. Assoc. J., 1970, 102, 846.

37. D. Grahame-Smith, J. Neurochem., 1971, 18, 1053.

38. D. F. Bogdanski, A. Pletscher, B. B. Brodie, and S. Udenfriend, J. Pharmacol. Exp. Ther., 1956, 117, 82.

39. J. Blundell, Neuropharmacology, 1984, 23, 1537.

40. G. L. Brown, Am. J. Psychiat., 1982, 139, 741.

41. F. Engbaek and B. Voldby, Clin. Chem., 1982, $28,624$. 
42. I. Hammel, Y. Naot, E. Ben-David, and H. Ginsburg, Anal. Biochem., 1978, 90, 840.

43. S. Baba, M. Utoh, M. Horie, and Y. Mori, J. Chromatogr. $B, \mathbf{1 9 8 4}, 307,1$.

44. T. Hayashi, M. Shimamura, F. Matsuda, Y. Minatogawa, H. Naruse, and Y. Iida, J. Chromatogr., B, 1986, 383, 259.

45. Z. D. Peterson, M. L. Lee, and S. W. Graves, J. Chromatogr., $B$, 2004, 810, 101

46. P. Tagari, D. Boullin, and C. Davies, Clin. Chem., 1984, 30
131.

47. C. G. Khatri and C. R. Rao, Sankhya Series A, 1968, 30, 167.

48. S. Torsten and G. W. Stewart, SIAM J. Numer. Anal., 1974, 11,61 .

49. E. Acar, D. M. Dunlavy, and T. G. Kolda, J. Chemom., 2011, 25, 67.

50. A. C. Olivieri and K. Faber, Anal. Chem., 2012, 84, 186. 